\title{
AN EMBEDDED DENSITY CONTROLLED TRAFFIC SYSTEM
}

\author{
Saneep K \\ Department of EEE \\ NSS College of Engineering \\ Palakkad, Kerala, India
}

\author{
Prasanth R K \\ Department of EEE \\ NSS College of Engineering \\ Palakkad, Kerala, India
}

\begin{abstract}
The paper is presented to develop a density based dynamic traffic signal system. The signal timing changes automatically on sensing the traffic density at the junction. Traffic congestion is a severe problem in many major cities across the world and it has become a nightmare for the commuters in these cities. Conventional traffic light system is based on fixed time concept allotted to each side of the junction which cannot be varied as per varying traffic density. Junction timings allotted are fixed. Sometimes higher traffic density at one side of the junction demands longer green time as compared to standard allotted time. The proposed system using a microcontroller of 8051 family duly interfaced with sensors, changes the junction timing automatically to accommodate movement of vehicles smoothly avoiding unnecessary waiting time at the junction. The sensors used in this project are IR and photodiodes are in line of sight configuration across the loads to detect the density at the traffic signal. The density of the vehicles is measured in three zones i.e., low, medium, high based on which timings are allotted accordingly. Further the project can be enhanced by synchronizing all the traffic junctions in the city by establishing a network among them. The network can be wired or wireless. This synchronization will greatly help in reducing traffic congestion.
\end{abstract}

Keywords - Embedded System, Traffic system, PIC16F877, Congestion Control

\section{INTRODUCTION}

The project is designed to develop a density based dynamic traffic signal system. The signal timing changes automatically on sensing the traffic density at the junction. Traffic congestion is a severe problem in many major cities across the world and it has become a nightmare for the commuters in these cities.

Conventional traffic light system is based on fixed time concept allotted to each side of the junction, which cannot be varied as per varying traffic density. Junction timings allotted are fixed. Sometimes higher traffic density at one side of the junction demands longer green time as compared to standard allotted time.
The proposed system using microcontroller family duly interfaced with sensors, changes the junction timing automatically to accommodate movement of vehicles smoothly avoiding unnecessary waiting time at the junction. The sensors used in this project are IR and photodiodes are in line of sight configuration across the loads to detect the density at the traffic signal. The density of the vehicles is measured in three zones i.e., low, medium, high based on which timings are allotted accordingly.

Further, the project can be enhanced by synchronizing all the traffic junctions in the city by establishing a network among them. The network can be wired or wireless. This synchronization will greatly help in reducing traffic congestion.

Public transport is the predominant mode of motorised local travel in cities. This is predominantly by road, since commuter rail services are available only in the seven metropolitan cities of Mumbai, Delhi, Chennai, Kolkata, Bengaluru, Kochi, Hyderabad and Pune, while dedicated city bus services are known to operate in at least 25 cities with a population of over one million. Intermediate public transport modes like tempos and cycle rickshaws assume importance in medium size cities. However, the share of buses is negligible in most Indian cities as compared to personalized vehicles, and two-wheelers and cars account for more than 80 percent of the vehicle population in most large cities.

Generally, traffic in most of the cities in India moves slowly, where traffic jams and accidents are very common, but in some cities like Chandigarh, wide roads and less vehicles contribute to lesser traffic. India has very poor records on road safely - around 90,000 people die from road accidents every year. At least 13 people die every hour in road accidents in the country, also in the year 2007 road accidents claimed more than 130,000 lives, overtaking China. A Reader's Digest study of traffic congestion in Asian cities ranked several Indian cities within the Top Ten for worst traffic.

In static traffic system the vehicles on a way will consumes only a fixed time. So, the traffic does not depends on the number of vehicles. It will consuming more time. 


\section{International Journal of Engineering Applied Sciences and Technology, 2020 \\ Vol. 4, Issue 9, ISSN No. 2455-2143, Pages 176-181 \\ Published Online January 2020 in IJEAST (http://www.ijeast.com)}

\section{EMBEDDED SYSTEM}

The power supply unit is used to provide a constant $5 \mathrm{~V}$ of DC supply from a $230 \mathrm{~V}$ of AC supply. These $5 \mathrm{~V}$ DC will acts as power to different standard circuits. It mainly uses 3 devices,

Bridge wave rectifier

Voltage regulator

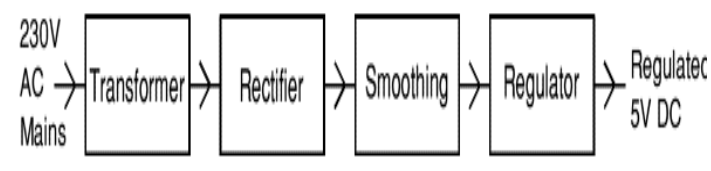

Fig. 1 Block Diagram of Power Supply

The microcontroller is Factory calibrated to $\pm 1 \%$. In this Software selectable frequency range of $8 \mathrm{MHz}$ to $32 \mathrm{kHz}$ and it also Software tunable. It specialized with Two-Speed StartUp mode, It Fail-safe clock monitoring for critical applications. It need clock mode switching during operation for low-power operation. The microcontroller is operates on Power-Saving Sleep mode. Power-on Reset (POR) and Selectable Brownout Reset (BOR) voltage. In-Circuit Debug (ICD) via two pins. It has High-endurance Flash/EEPROM cell, 100,000 erase/write cycle enhanced Flash program memory, typical, 1,000,000 erase/write cycle data EEPROM memory, It's typical Data EEPROM retention is greater than 40years. It can Self-reprogrammable under software control. It has Programmable code protection. It has 1 input only pin and $36 \mathrm{I} / \mathrm{O}$ pins. It has high sink/source current $25 \mathrm{~mA}$. It has a facility to interrupt-on-pin change option. It uses TMR0,8-bit timer/counter with 8-bit prescaler, TMR1 enhanced And 16bit timer/counter with prescaler,It resembles External Gate Input mode and dedicated low-power $32 \mathrm{kHz}$ oscillator. It has TMR2 also with 8-bit timer/counter with 8-bit period register, prescaler and postscaler. It have a good Analog Features.It contains 10-bit 14 channel Analog-to-Digital (A/D) Converter and 2 Analog Comparator modules withProgrammable onchip Voltage Reference (CVREF) module (\% of VDD) and Fixed 0.6 Vref. The Comparator inputs and outputs are externally accessible.

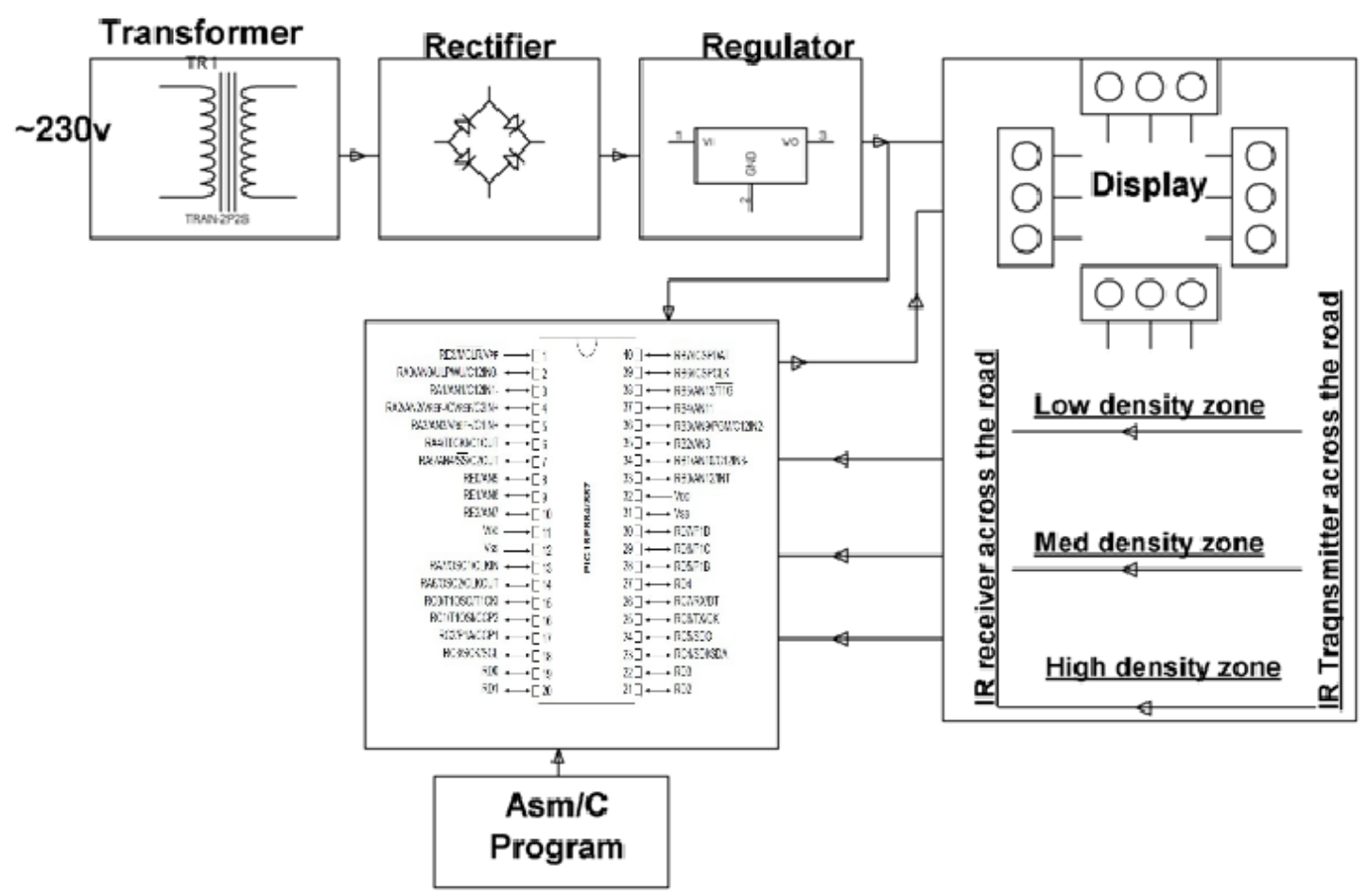

Fig. 2 Block Diagram of Proposed System 


\section{A. IR LED}

Such LEDs are usually made of gallium arsenide or aluminum gallium arsenide. They, along with IR receivers, are commonly used as sensors. The appearance is same as a common LED. Since the human eye cannot see the infrared radiations, it is not possible fan IR LED, also known as IR transmitter, is a special purpose LED that transmits infrared rays in the range of $760 \mathrm{~nm}$ wavelength, or a person to identify whether the IR LED is working or not, unlike a common LED. To overcome this problem, the camera on a cell phone can be used. The camera can show us the IR rays being emanated from the IR LED in a circuit.

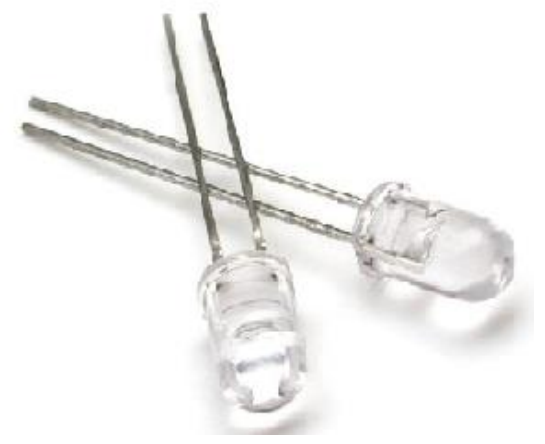

Fig. 3 IR LED

\section{B. Photo Diode}

A photodiode is a type of photo detector capable of converting light into either current or voltage, depending upon the mode of operation. Photodiodes are similar to regular semiconductor diodes except that they may be either exposed (to detect vacuum UV or X-rays) or packaged with a window or optical fibred connection to allow light to reach the sensitive part of the device.

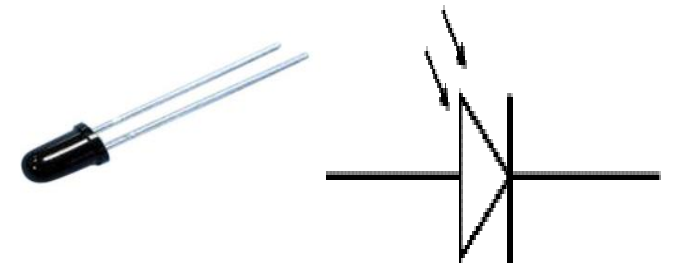

Fig. 4 Photo Diode

\section{Transistor}

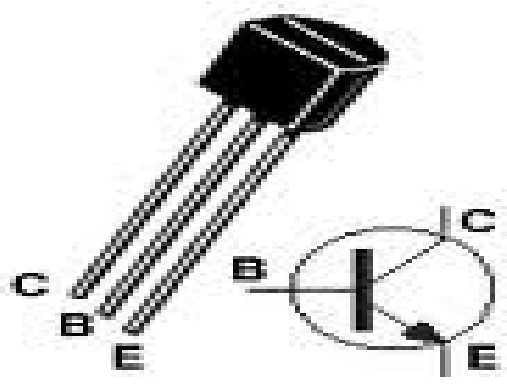

Fig. 5 Transistor

The BC547 transistor is an NPN Epitaxial Silicon Transistor. The BC547 transistor is a general-purpose transistor in small plastic packages. It is used in general-purpose switching and amplification BC847/BC547 series $45 \mathrm{~V}$, $100 \mathrm{~mA}$ NPN general-purpose transistors. Whenever base is high, then current starts flowing through base and emitter and after that only current will pass from collector to emitter.

\section{CIRCUIT DiagRAM}

The project uses the IR interruption concept for generating logic states to the input of the MC. To achieve the same a number of IR diodes are used facings photodiodes. While the IR light falls on the photodiode the resistance of the photodiode falls increasing the bias voltage. Logic high sensed by the MC input changes the green $\mathrm{ON}$ time to a higher value for allowing more vehicles to pass through. After sometime in case any other way gets more logic high, the sequential timing gets automatically increased for that way. Based on the IR interruption the green ON time increases, thus more the vehicle longer will be the green signal time. Thus dynamic time control is achieved based on the traffic density. In the case of emergency vehicles like ambulance, we provide a RF remote. It act as RF transmitter and a RF receiver will provided on the traffic system. Whenever the vehicle comes the remote should be pressed. Hence the system identifies the line where the ambulance is present, and then system gives more priority to that line. After passing the ambulance the system continue with earlier timing and setting. Thereby we can control the traffic congestion and also it is utilizes maximum time with usage, i.e. no wastage of time. 

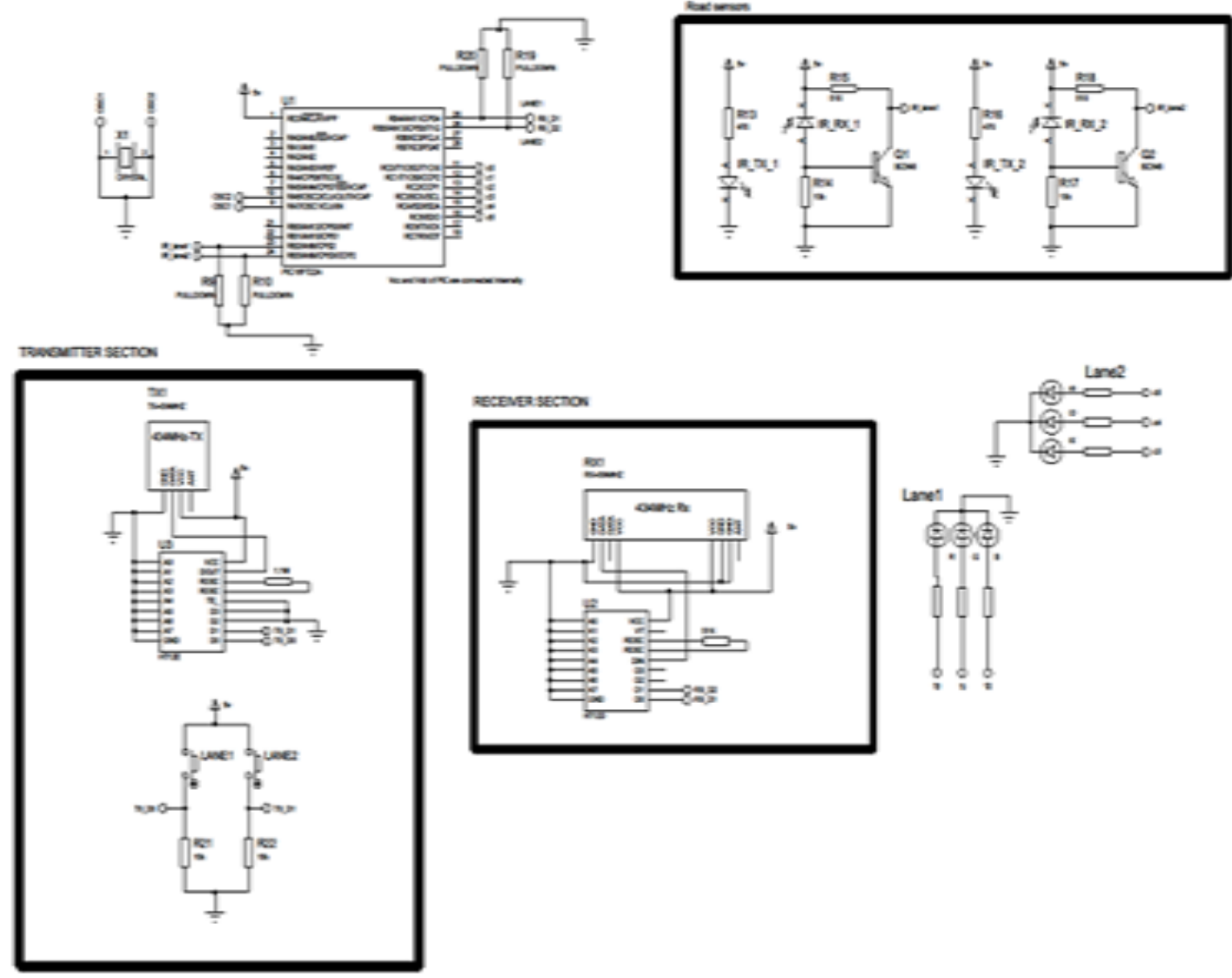

Fig. 6 Circuit Diagram

\section{HARDWARE IMPLEMENTATION}

The proposed system of dynamic traffic controlling is design as per the figure. Our system of project consists of LED signal systems for two paths, two pair of IR sensors. One pair of IR sensor consists of one IR transmitter and One IR receiver. Here the system is proposed for two path junction. i.e. two lines. Line 1, Line 2.

In the case of the normal traffic system, the green light for the line- 1 and line- 2 are fixed and same. So, when a line not having any vehicle then only the green light will glow the same time that glow for the line-2.Here a dynamic traffic system that controls the time for green light is introduced by considering the density of vehicles in the line. Now consider the line-1 will contain more vehicle than line-2, then the system provide more time for green light corresponds to line 1 and less time for line-2. And vice versa.

This proposed system gives more priority in the case of emergency vehicle like ambulance. For this process, a pair of IR transmitter and receiver is installed. In the ambulance the transmitter is placed, and the receiver is placed along with the traffic signal system arrangements. So, the ambulance comes through the line-1 then press the transmitter switch for line-1. At that moment the signal system stops and gives green signal for the line-1.after passing the ambulance from the junction the transmitter switch again pres. Then the system continues to work properly from where the system stops. In the hardware modelling, a separate remote is provided for the ambulance. 
International Journal of Engineering Applied Sciences and Technology, 2020

Vol. 4, Issue 9, ISSN No. 2455-2143, Pages 176-181

Published Online January 2020 in IJEAST (http://www.ijeast.com)

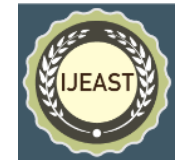

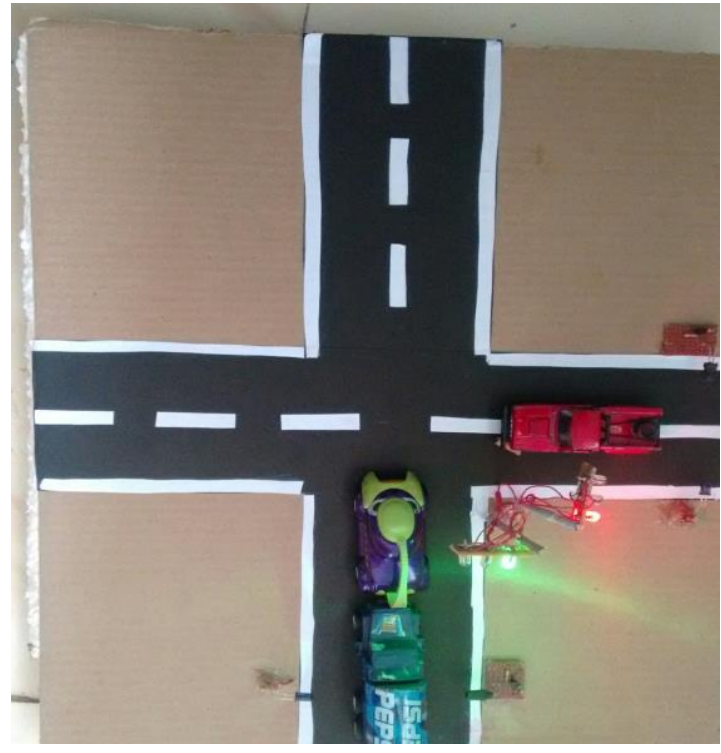

Fig. 7 Hardware Implementation

\section{CONCLUSION}

The progress in science \& technology is a nonstop process. New things and new technology are being invented. As the technology grows day by day, we can imagine about the future in which thing we may occupy every place. The proposed system based on PIC microcontroller is found to be more compact, user friendly and less complex, which can readily be used in order to perform. Several tedious and repetitive tasks. However, it is designed keeping in mind about the need for industry. Due to the probability of high technology used this project is fully software controlled with less hardware circuit. The feature makes this system is the base for future systems. The proposed system will make the industries partially automated and which helps the operator to identify the fault easily. These features are improves the working of an industry without making time delay to solve the faults occurring in the electrical machines. The principle of the development of science is that "nothing is impossible". So we shall look forward to a bright \& sophisticated world.

\section{REFERENCES}

[1] Vivek, Tyagi, Senior member IEEE, (2012) "Vehicular Traffic Density State Estimation Based On Cumulative Road Acoustics" in
IEEE Transaction on Intelligent Transportation System. Vol. 23 No. 3 .

[2] D.Manoj (2012) "Density Based Traffic Control System" Electrical \&Electronics Engineering Department mahatma Gandhi institute of technology.

[3] S. Theodore, Rappaport, (2011) "Wireless Communications Principles and Practices",second edition.

[4] B. Prashanth Kumar, B. Karthik,(2011) "micro controller based traffic light controller" , department of Electrical \&Electronics Engineering, Gokaraju Rangaraju institute of engineering \& technology.

[5] Sabya sanchi kanojia, (2012) "Real -time Traffic light control and Congestion avoidance system", International Journal of Engineering Research and Applications (IJERA), pp.925-929, Vol. 2, Issue 2,

[6] J. Clerk Maxwell, "A Treatise on Electricity and Magnetism", 3rd ed., vol. 2. Oxford: Clarendon, 1892, pp.68-73.

[7] Bilal Ghazal, Khaled EIKhatib, Khaled Chahine, Mohamad Kherfan, (2016) "Smart traffic Light Control System", IEEE.

[8] Chandana K K, Dr. S. Meenakshi Sundaram, Cyana D'sa, Meghana N Swamy, Navya K, (2017) "A Smart Traffic Management System for Congestion Control and Warnings Using Internet of Things (IoT)", Saudi Journal of Engineering and Technology, Vol-2, Issue 5:192-196.

[9] MihaelaCondurat, Alina Mihaela Nicuţă, RaduAndrei, (2017) "Environmental Impact of Road Transport Traffic. A Case Study for County of Iaşi Road Network", 10th International Conference Interdisciplinarity in Engineering, Procedia Engineering, Volume 181, Pages 123-130.

[10] H. O. Al-Sakran, (2016) "Intelligent traffic information system based on the integration 
of Internet of Things and Agent technology," International Journal of Advanced Computer Science and Applications (IJACSA), vol. 6, no. 2, pp. 37-43, 2016.

[11] ] Priyanka Khanke, Prof. P. S. Kulkarni ,(2014) "A Technique on Road Tranc Analysis using Image Processing", Vol. 3 Issue 2.

[12] Jie Zhou.et al. (2007) "Moving Vehicle Detection for Automatic Traffic Monitoring" IEEE Transactions on Vehicular Technology, Vol. 56, No. 1.

[13] Wisnu Jatmiko, et.al, (2010) " Detection and Counting of Vehicles Based on Video Processing In Distributed Traffic System", International Conference on Advance Computer Science and Information System 
International Journal of Engineering Applied Sciences and Technology, 2020 Vol. 4, Issue 9, ISSN No. 2455-2143, Pages 176-181

Published Online January 2020 in IJEAST (http://www.ijeast.com) 